\title{
Implementación de un servicio de referencia virtual por chat en una biblioteca universitaria argentina
}

\author{
Marina Borrell${ }^{\star}$, Evangelina Maciel ${ }^{\star}$
}

Resumen: En el presente trabajo se comentará la experiencia de la biblioteca Joaquín V. González de la facultad de Ciencias Jurídicas y Sociales de la Universidad Nacional de La Plata (Argentina) en la implementación y puesta en marcha de un servicio de referencia virtual vía chat, para responder consultas en tiempo real, destinado a la comunidad universitaria.

Palabras clave: servicio de referencia, referencia virtual, chat, biblioteca universitaria, Argentina.

\section{Implementation of a virtual reference service by chat in an Argentinian academic library}

\begin{abstract}
The following article will comment the experience of the library Joaquin $V$. González of the Facultad de Ciencias Juridicas y Sociales of the Universidad Nacional de La Plata (Argentina) in the implementation of a virtual reference service by chat, to answer requests in real time, directed to the university community
\end{abstract}

Keywords: reference service, virtual reference, chat, universitary library, Argentina.

\section{Algunos conceptos sobre referencia virtual}

El servicio de referencia tradicionalmente fue uno de los servicios más importantes que brindaban las bibliotecas, a través de él se podía tener acceso a la colección, consultar una fecha, un dato histórico, obtener una bibliografía y mucho más. (Melnik, 2005) La referencia se ofrecía en la propia biblioteca por lo que se contaba con un escritorio o mostrador en el que un referencista recibía a los usuarios y los orientaba en su búsqueda de información.

Con el correr del tiempo y el surgimiento de tecnologías de información avanzada, este servicio fue evolucionando hasta llegar a lo que hoy damos en llamar servicio de referencia virtual. Es decir, ofrecerle información al usuario sin que tenga que desplazarse hasta la biblioteca, esto puede hacerlo a través de las distintas opciones de referencia virtual tales como mail, formularios electrónicos o chat, entre los más difundidos.

* Facultad de Ciencias Jurídicas y Sociales. Biblioteca Joaquín González. Universidad Nacional de la Plata. Argentina. Correo-e: referenciavirtual@jursoc.unlp.edu.ar

Recibido: 22-12-08; 2. ${ }^{a}$ versión: 15-4-09. 
Este nuevo servicio ha generado una serie de discusiones por parte de los bibliotecarios que no logran ponerse de acuerdo en la unificación de su denominación, es por esto que utilizan de forma indistinta términos tales como: referencia virtual, referencia en tiempo real, referencia digital, e-referencia, referencia en línea o bien sus equivalentes en inglés. (Zanin Yost, 2004)

\section{Servicio de referencia por chat en la Biblioteca Joaquín V. González}

La biblioteca Joaquín V. González pertenece a la facultad de Ciencias Jurídicas y Sociales de la Universidad Nacional de La Plata-Argentina. La biblioteca fue fundada el 7 de junio de 1906. Su fondo bibliográfico tuvo como base la donación de 4.450 volúmenes que efectuó quien fuera Rector de la Universidad, el Dr. Joaquín Víctor González.

La Biblioteca universitaria es una unidad funcional de apoyo al estudio, a la docencia y a la investigación. En la actualidad cuenta con un fondo documental de alrededor de 41.480 volúmenes y 1.030 títulos de publicaciones periódicas. Entre sus socios podemos contar 8.000 alumnos de grado, 104 de posgrado, 113 docentes e investigadores, y 109 socios de otras Instituciones que poseen convenios con la Facultad.

Cuenta con servicios para incentivar al alumno en el uso de la biblioteca, entre los que podemos mencionar, la renovación telefónica de libros, digitalización de material antiguo, servicio de reprografía, formación de usuarios, capacitaciones en el manejo de fuentes jurídicas en línea, entre otros.

Todos los servicios pueden consultarse en la página web de la biblioteca: http://der.jursoc.unlp.edu.ar/contenido.php?tipo=4

\section{Inicio del proyecto}

A principios del año 2006 y debido a los cambios producidos en el edificio de la biblioteca y al interés por parte de las autoridades de ofrecer a toda la comunidad educativa nuevos servicios virtuales, se comienza a pensar en ofrecer un servicio de referencia virtual por chat.

Como primera tarea se convocó al área Informática de la facultad para que diseñara un software que permitiera ofrecer desde la página Web de la biblioteca un servicio de referencia por chat a los alumnos, docentes, investigadores y personal de la facultad, indistintamente de que fueran socios o no. Se tuvo en cuenta para el diseño las recomendaciones de la IFLA para la creación y mantenimiento de servicios de referencia, tales como: elaborar objetivos concretos y realizables; definir un grupo básico de usuarios; elaborar una estructura y diseño estándares, y utilizarlos de un modo coherente a lo largo de todas las páginas, entre otros. (IFLA, 2003) 
Entre sus características debía contar con la particularidad de ser no descargable, esto implicaba que los usuarios no tuvieran que bajarse ningún programa a su ordenador para poder ingresar al chat. Una vez diseñada esta funcionalidad y avanzado el desarrollo de la misma se convocó a las bibliotecarias que se ocuparían del chat para realizar una primera prueba piloto y comprobar su funcionamiento.

Esta primera versión arrojó varios errores de funcionamiento, entre los que se contaba la imposibilidad de restringir la cantidad de usuarios que ingresaban, la falta de un alerta sonoro para que le indicara al bibliotecario cuando ingresaba un usuario al servicio, y algunas cuestiones de diseño. Una vez resueltos los cambios necesarios se volvió a testear la plataforma comprobando su buen funcionamiento y se comenzó a trabajar en el lanzamiento del servicio.

\section{Puesta en marcha}

Con el objeto de difundir el nuevo servicio entre todos los integrantes de la comunidad educativa de la Facultad y poner en su conocimiento el funcionamiento del chat, se decidió realizar una serie de actividades que permitieran darle la difusión necesaria entre los futuros usuarios; entre ellas podemos citar:

- Envío de mails a docentes y alumnos en los que se comentaba la inminente apertura del servicio de referencia por chat de la biblioteca.

- Presentación de la Ponencia "Biblioteca Centenaria de cara al futuro" en las 5. Jornadas Expo Universidad Comunidad 2006-La Plata en el Pasaje Dardo Rocha, donde se exponía la finalidad y funcionamiento del servicio de referencia virtual.

- Presentación y demostración del servicio; ante las autoridades de la facultad y público en general en la sala de lectura de la biblioteca.

La puesta en marcha del servicio se realizó el día 26 de octubre de 2006 en el marco de los actos por el centenario de la biblioteca Joaquín V. González. Allí se realizó una demostración del funcionamiento frente a las autoridades de la facultad, al personal de la biblioteca, alumnos, docentes y público en general.

El acto inaugural fue muy bien recibido por todos los asistentes ya que las bibliotecas universitarias argentinas no suelen contar con este tipo de servicio, siendo ésta unidad académica una de las pioneras en implementarlo, generando así una imagen positiva tanto hacia los usuarios como hacia la comunidad bibliotecaria en general.

\section{Funcionamiento del servicio}

Cuando se diseñó el software para el chat, se pensó en realizar un producto que fuera intuitivo en el uso y con ciertas similitudes a los programas comercia- 
les de mensajería instantánea para que al usuario le resultara fácil de utilizar en un entorno amigable.

Para comenzar a usar el chat hay que ingresar a la web de la biblioteca, allí en el apartado Consultas on-line vía chat se puede observar la página de ingreso al servicio, en la que constan el horario de atención, los destinatarios y el mail de consulta; también se incluyó una guía de uso con respuestas sobre que tipo de material se puede solicitar, que tipo de información no se brinda y unas breves pautas de comportamiento.

Si bien este servicio lo ofrece la biblioteca, no es requisito ser socio de la misma para poder utilizarlo, ya que se trabaja en coordinación con el sistema de gestión de alumnos (SIU-Guaraní) que posee el departamento de alumnos, la base de datos de docentes y personal administrativo de la Facultad y la coordinación del Área de informática que logró que todos los miembros de la Institución en general puedan acceder al servicio, indistintamente de que estén asociados o no a la Biblioteca basta con poseer un lazo de pertenencia a la Institución.

Aquellos usuarios que deseen ingresar, deben escribir su número de DNI (Documento Nacional de Identidad), sin puntos, en el casillero correspondiente, y presionar el botón ingresar. Una vez dentro del servicio se pueden observar los datos completos del alumno que se conectó (legajo ${ }^{1}$, apellidos y nombre) en el caso de docentes y personal se visualiza el número de DNI y el apellido y nombre. Figura una referencia a la cantidad de usuarios conectados, no puede haber más de dos por vez, y la opción de descargar la Guía de usuarios. Más abajo figura una pequeña pantalla para ir leyendo el dialogo que se mantiene con el bibliotecario y un casillero para escribir las consultas. Véase la Figura 1.

En cuanto a la pantalla del referencista, es muy similar a la del usuario, solo que permite también, habilitar el uso de una cámara Web, para que el usuario pueda ver al bibliotecario de turno; y un alerta sonoro, que se activa cuando una persona ingresa al chat para que el bibliotecario pueda realizar otras tareas sin desatender el servicio ni estar pendiente de observar la ventana de conexión. Véase la Figura 2.

En ambas pantallas figura debajo un listado de preguntas frecuentes, sobre temas recurrentes (FAQ), por ejemplo: ¿Que tengo que presentar para hacerme socio?, ¿cual es el horario de la biblioteca?, ¿cuantos libros me puedo llevar?, etc.

El chat está pensado para ofrecerle al usuario respuestas al instante, para evitar que tenga que desplazarse hasta la biblioteca para obtener material que se encuentra en formato digital y para asesorar a los usuarios en la búsqueda de material. (Rodríguez Briz, 2005)

Entre las principales consultas que se reciben podemos mencionar la solicitud de jurisprudencia; en general los alumnos solicitan un fallo ${ }^{2}$ específico que les

\footnotetext{
1 Número interno otorgado a cada alumno que pertenece a la Facultad.

2 Es un acto procesal emanado de un órgano jurisdiccional que decide la causa o un punto sometido a su conocimiento (Osorio, 1996).
} 
FIGURA 1

Pantalla del usuario

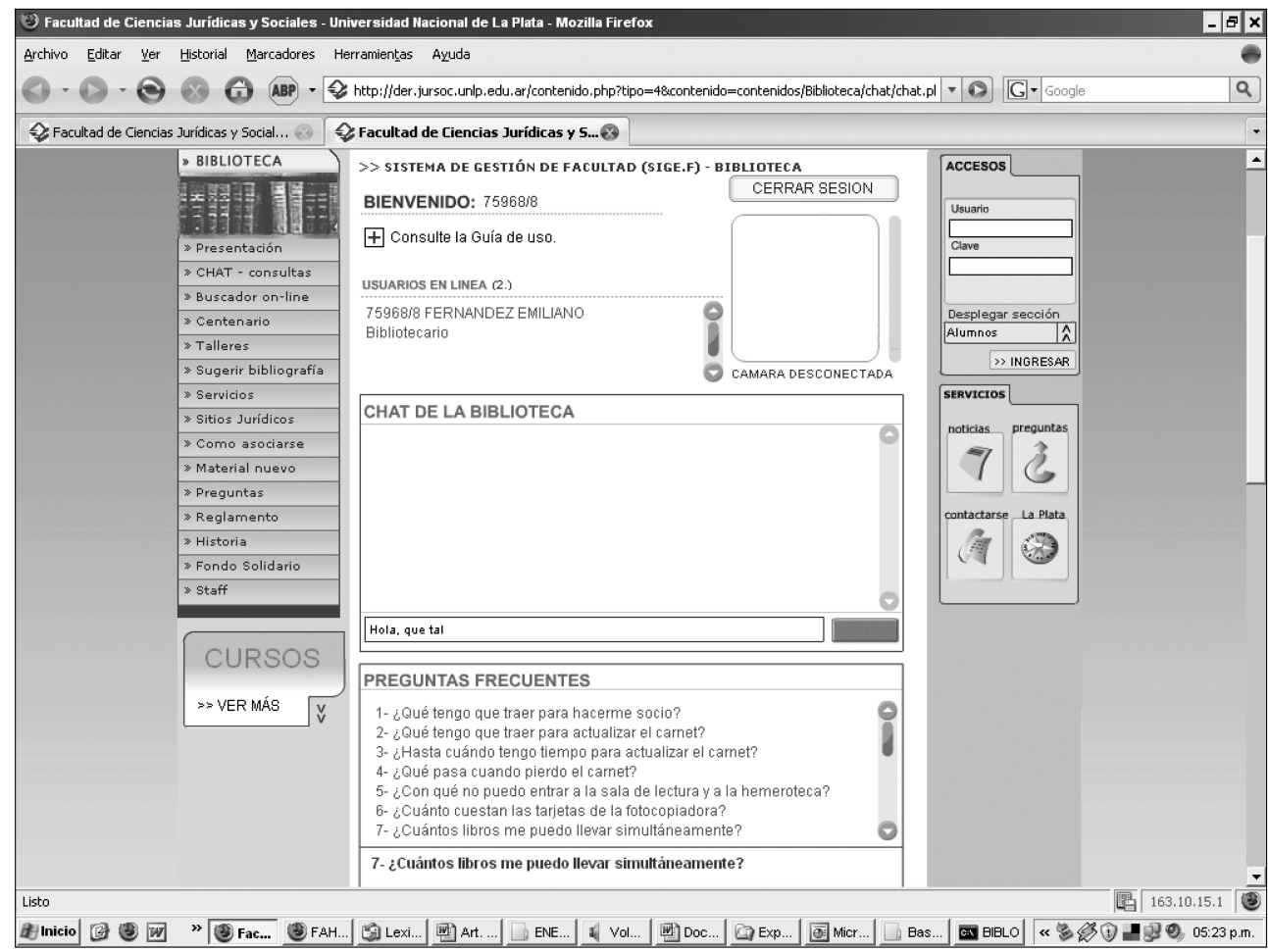

dio el docente en clase o que figura en la bibliografía de la cátedra, o bien jurisprudencia sobre un tema en particular; en cualquiera de los casos se busca el material en bases de datos comerciales o gubernamentales y se le envía al usuario el texto completo por mail. También suelen consultar por legislación, aunque en menor medida, ya el país cuenta con bases de datos gubernamentales de acceso abierto que cuentan con legislación actualizada.

Otro tipo de consulta que suele presentarse es la relacionada a material docente; tanto de artículos de revista a texto completo digitales; como la existencia de determinados títulos en el fondo bibliográfico, en este último caso se consulta el catálogo, se muestra, a través de la ventana del chat, la cita bibliográfica con su respectiva signatura topográfica y la tabla de contenido de la obra, para que el usuario pueda venir luego a retirar el libro en préstamo o a consultarlo en la sala de lectura. En cuanto a los artículos de publicaciones, se buscan en las bases de datos a las que está suscrita la biblioteca o en la web de la propia revista y se le envía al usuario el material por mail o los datos referidos al tomo, fascículo o número de la publicación periódica en papel en el que podrá consultar el texto. 
FIGURA 2

\section{Pantalla del bibliotecario}

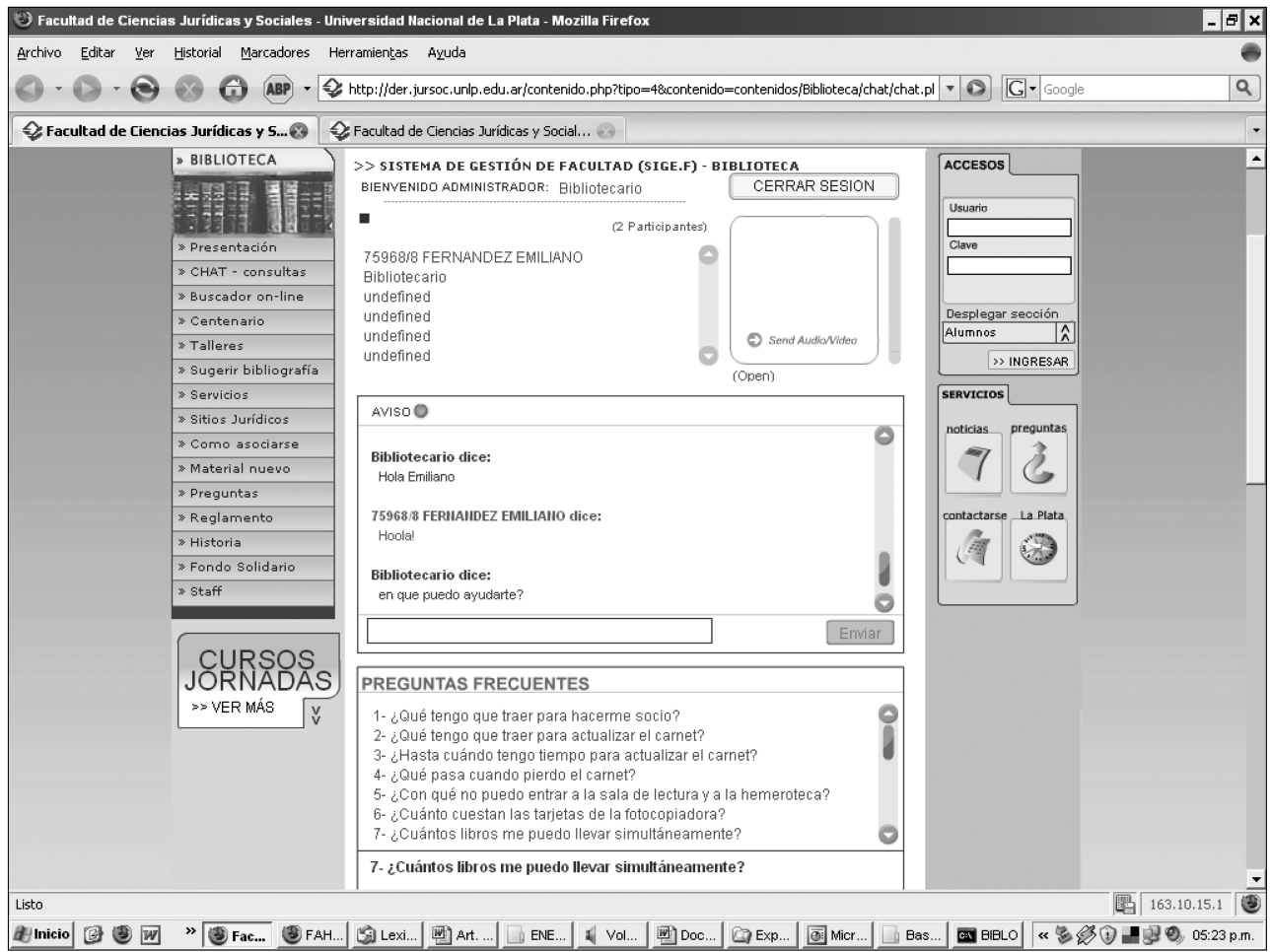

Y por último se ofrece información administrativa de la biblioteca (horarios, número de teléfono, cursos que se dictan, etc.) y asesoramiento en el uso del catálogo web y recursos electrónicos disponibles en la página de la biblioteca.

Con el objeto de mantener un criterio uniforme a la hora de responder consultas se establecieron cuales serían los puntos en los que no se brindaría información a través del chat y también se implementó un máximo de diez documentos que se envían por sesión a cada usuario. Debido a los diferentes tipos de préstamo (normales, especiales de fin de semana y nocturnos) que ofrece la biblioteca y al sistema de reserva que utiliza, se decidió no informar sobre que libros tiene un usuario en préstamo y cuando le vencen, ni realizar renovaciones de los mismos. Tampoco información referida a sanciones que le fueron aplicadas, ya que para tal fin deben acercarse personalmente al mostrador de préstamos.

Posteriormente, este servicio se amplió a toda la red de bibliotecas pertenecientes a la universidad. A éstas se les otorgó una clave de acceso que consiste en un número alfanumérico coincidente con las siglas oficiales brindadas por el CAICyT (Centro Argentino de Información Científica y Técnica) para cada biblio- 
teca. En la reunión de directores de bibliotecas de la UNLP se le entregó a cada director la clave de acceso para que la hiciera extensiva a su personal del área de referencia, para que pudieran utilizar el servicio con los mismos beneficios y obligaciones que los usuarios de la facultad.

\section{Tareas bibliotecológicas}

Con el fin de poder establecer el uso del servicio y poder realizar futuras comparaciones, se confeccionó una planilla de cálculo para el registro de datos de los usuarios que ingresan al servicio para realizar estadísticas.

Los datos que se volcaron a la planilla fueron los siguientes:

Fecha: Día de atención del servicio.

Tipo de usuario: Alumno, Docente/Investigador, Empleado

Datos del usuario: Apellido y Nombres

Tipo de consulta: Información general, Existencia de documento, Legislación, Jurisprudencia, Doctrina

Turno: Mañana, Tarde

Bibliotecario: Nombre del bibliotecario que atendió la consulta.

Como establece Wasik son los datos estadísticos los que nos permiten evaluar el chat para comprobar su desarrollo y evolución y poder seguir así ofreciendo un servicio de calidad.

Las estadísticas tomadas hasta el momento nos permiten visualizar la curva de crecimiento que ha tenido el servicio desde octubre de 2006 hasta la fecha, gracias a las distintas tareas tendientes a darle difusión entre las que se puede mencionar la demostración que se hace del chat en los cursos de formación de usuarios de la biblioteca.

En la Figura 3 podemos observar que entre los meses de febrero a diciembre (en los que el chat se encuentra abierto), la curva de crecimiento va en ascenso en lo referente a la cantidad de alumnos que se conectan. Con respecto a los docentes podemos observar en la Figura 4 el poco uso que hicieron del servicio durante 2006 y 2007, dado lo acotado del tiempo de apertura no podemos establecer cual es el factor que hace que los docentes le den poco uso a esta herramienta, aunque a partir de 2008 la tendencia pareciera mejorar.

Los usuarios del chat han presentado dos tipos diferenciados de consultas a lo largo de los años, por un lado, aquellas que solo requieren de un dato puntual, por ejemplo el número de teléfono de la Biblioteca; o la existencia de un documento, como por ejemplo un libro en particular; y por otro lado aquella que requiere el envío de material por mail, como una ley, una artículo de una revista o un fallo. 
FIGURA 3

Cantidad de alumnos conectados

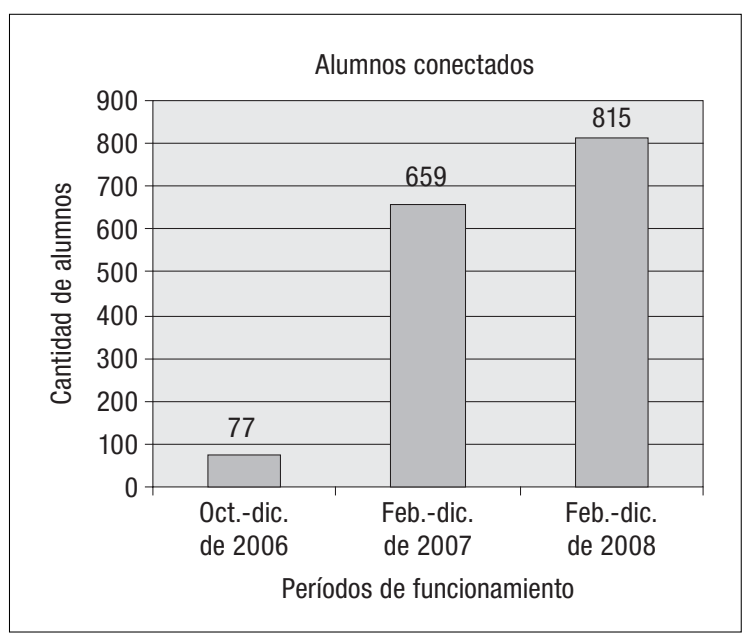

FIGURA 4

\section{Cantidad de docentes conectados}

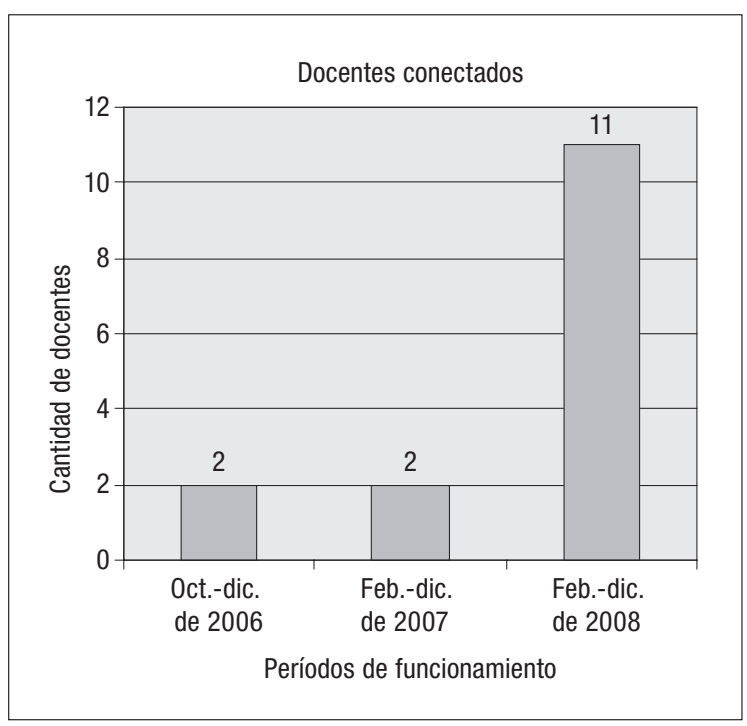

En cuanto al primer tipo de consultas, las preguntas más frecuentes son: horarios de apertura y cierre de los servicios, el número de teléfono para renovar los libros, fechas de los cursos que brinda la biblioteca, entre otras y además si la biblioteca cuenta con algún libro puntual. 
La baja en consultas registrada en 2008 puede deberse a que el servicio de formación de usuarios de la Biblioteca se ocupa de brindar toda la información general necesaria y los alumnos ya no requieren recibirla a través del chat.

En la Tabla I puede comprobarse que mientras en 2007 se realizaron 104 consultas sobre algún dato general de la Biblioteca estas consultas descendieron a 90 en el 2008, lo mismo sucede con las preguntas referidas a la existencia o no de un determinado libro en el fondo bibliográfico de la Biblioteca, cuando en 2007 las consultas fueron 85 este número descendió considerablemente en 2008 con apenas 55 consultas.

\section{TABLA I}

Tipos de consultas

\begin{tabular}{l|c|c|c|c}
\hline & $\begin{array}{c}\text { Octubre- } \\
\text { diciembre } \\
\text { de 2006 }\end{array}$ & $\begin{array}{c}\text { Febrero- } \\
\text { diciembre } \\
\text { de 2007 }\end{array}$ & $\begin{array}{c}\text { Febrero- } \\
\text { diciembre } \\
\text { de 2008 }\end{array}$ & Total \\
\hline Información general & 25 & 104 & 90 & $\mathbf{2 1 9}$ \\
\hline Existencia de documento & 22 & 85 & 55 & $\mathbf{1 6 2}$ \\
\hline
\end{tabular}

En cuanto al segundo tipo de consulta la mayoría corresponden a la solicitud de jurisprudencia, es decir, un fallo puntual de un tribunal argentino, estos pedidos se duplicaron de 2007 a 2008; también hay un importante crecimiento en el envío de artículos de doctrina escritos por reconocidos profesionales y, por último, y en menor medida, la solicitud de leyes y decretos, esto se debe a que el país cuenta con diferentes sitios de acceso público para buscar este tipo de información, mientras que la doctrina y la jurisprudencia se encuentra en bases de datos a las que la biblioteca está suscripta. Véase Tabla II

TABLA II

Archivos enviados

\begin{tabular}{l|c|c|c|c}
\hline & $\begin{array}{c}\text { Octubre- } \\
\text { diciembre } \\
\text { de 2006 }\end{array}$ & $\begin{array}{c}\text { Febrero- } \\
\text { diciembre } \\
\text { de 2007 }\end{array}$ & $\begin{array}{c}\text { Febrero- } \\
\text { diciembre } \\
\text { de 2008 }\end{array}$ & Total \\
\hline Legislación & 0 & 41 & 47 & $\mathbf{8 8}$ \\
\hline Jurisprudencia & 24 & 202 & 413 & $\mathbf{6 3 9}$ \\
\hline Doctrina & 28 & 67 & 134 & $\mathbf{2 2 9}$ \\
\hline
\end{tabular}

También realizamos un backup diario de las conversaciones mantenidas con los usuarios, para poder llevar un registro de las mismas y en caso de que un alumno nos solicite su sesión de chat, poder brindarle una copia. 
Uno de los puntos importantes de la implementación de este servicio es el que nos permite detectar material faltante en nuestra colección o propuestas a nuevas suscripciones, ya que muchos usuarios se conectan para consultar por un libro nuevo o material jurisprudencial que el docente mencionó en la cátedra y que no llegó a incluir en el programa, es así como tomamos conocimiento del material y lo informamos al área de desarrollo de colección.

\section{Ventajas y desventajas}

Ofrecer un servicio de referencia virtual por chat en una biblioteca universitaria es todo un desafío, es por ello que hay que tener muy en cuenta las ventajas y desventajas que este tipo de servicio trae, aparejadas. Entre una de sus principales ventajas contamos con la inmediatez de la respuesta, lo que evita la demora de información para el usuario; también el hecho de que no tenga que desplazarse hasta la Biblioteca para aclarar una duda; el mayor acercamiento que permite al público joven y la posibilidad de brindarle un servicio de calidad para estudiantes no residentes en nuestra ciudad, dado que ésta facultad cuenta con la posibilidad de rendir las materias en forma libre (por medio de un examen sin necesidad de cursar).

Entre sus desventajas debemos mencionar la posibilidad de que se nos hagan preguntas triviales, que nada tienen que ver con la finalidad del servicio, la pérdida del "cara a cara" con el usuario; que los adultos consideren el chat poco serio, y las limitaciones de expresarse en pequeños textos escritos. También el usuario actual tiene el "vicio" de considerar que todo se encuentra en la red, cuando existe cierta información con cierta antigüedad que no está en formato digital, esto podría decirse que genera poco éxito en la respuesta y la posibilidad de que el mismo no se vuelva a conectar o que desconfíe de la búsqueda realizada por el bibliotecario.

\section{Conclusiones}

Finalmente queremos mencionar el desafío que significó poner en marcha un servicio de esta magnitud en una biblioteca universitaria, con todas sus ventajas y desventajas y el desafío que significa día a día poder acercar información al usuario en el momento en que la necesita.

Si bien hay consultas que exceden las posibilidades del chat, otras se solucionan a la brevedad y le evitan al usuario el tener que acercarse a la Biblioteca para obtener respuesta a alguna duda simple, enviarle el fallo que necesita leer o informarle que el libro que le recomendaron se encuentra en la biblioteca le permite organizar sus tiempos de otra forma y hacer rendir mejor sus horas de estudio. También, por parte de la biblioteca, captar nuevos usuarios al contar con un servicio de última generación y poder renovar el fondo bibliográfico gra- 
cias a las sugerencias de material por parte de la comunidad que utiliza este servicio.

A nosotras como bibliotecarias, el chat nos da la posibilidad de formarnos en una nueva tarea, la de desarrollar competencias para un nuevo servicio y de interactuar de forma diferente, pero no por ello menos importante, con los usuarios.

\section{Bibliografia:}

Estevez, A. (2005): Servicio de referencia digital. Absysnet, Disponible en: http://www. absysnet.com/tema/tema43.html. [Fecha de consulta: 18/11/08].

IFLA (2006): Recomendaciones para el servicio de referencia digital. IFLANET, Disponible en: http://www.ifla.org/VII/s36/pubs/drg03-s.htm. [Fecha de consulta: 16/12/08].

Melnik, D. (2005): Principios de referencia: fuentes y servicios. Buenos Aires; Alfagrama, p. 160 .

Merlo Vega, J. A. (2005): Servicios públicos de referencia en línea. BiD: textos universitaris de Biblioteconomia i Documentació, jun (14).

Disponible en: http://www.ub.es/bid/14merlo2.htm [Fecha de consulta: 4/11/08].

Osorio, M. (1996): Diccionario de ciencias jurídicas, politicas y sociales. (26. ${ }^{a}$ ed.) Buenos Aires; Heliasta, p. 1038.

Rodríguez Briz, F. (2005): Los servicios de referencia virtual: Surgimiento, desarrollo y perspectivas a futuro. Buenos Aires; Alfagrama, p. 156.

Wasik, J. Building and Maintaining Digital Reference Services. ERIC Digest (1999): Disponible en: http://www.eric.ed.gov/ERICWebPortal/custom/portlets/recordDetails/detailmini.jsp?_nfpb=true\&_\&ERICExtSearch_SearchValue_0=ED427794\&ERICExtSearch_ SearchType_0=eric_accno\&accno=ED 427794 [Fecha de consulta: 12/11/08].

Zanin Yost, A. (2004): Digital Reference. What the Past has Taught Us and What the Future Will Hold. Library Philosophy and Practice, vol. 7 (1). Disponible en: http://www. webpages.uidaho.edu/ mbolin/zanin-yost.htm [Fecha de consulta: 5/12/08]. 
